\title{
Accurate, Fast Fall Detection Using Gyroscopes and Accelerometer-Derived Posture Information
}

\author{
Qiang Li, John A. Stankovic, Mark Hanson, Adam Barth, John Lach \\ University of Virginia \\ \{lq7c, stankovic, mah6s, atb4c, jlach\}@virginia.edu \\ Gang Zhou \\ College of William and Mary \\ gzhou@cs.wm.edu
}

\begin{abstract}
Falls are dangerous for the aged population as they can adversely affect health. Therefore, many fall detection systems have been developed. However, prevalent methods only use accelerometers to isolate falls from activities of daily living (ADL). This makes it difficult to distinguish real falls from certain fall-like activities such as sitting down quickly and jumping, resulting in many false positives. Body orientation is also used as a means of detecting falls, but it is not very useful when the ending position is not horizontal, e.g. falls happen on stairs.

In this paper we present a novel fall detection system using both accelerometers and gyroscopes. We divide human activities into two categories: static postures and dynamic transitions. By using two tri-axial accelerometers at separate body locations, our system can recognize four kinds of static postures: standing, bending, sitting, and lying. Motions between these static postures are considered as dynamic transitions. Linear acceleration and angular velocity are measured to determine whether motion transitions are intentional. If the transition before a lying posture is not intentional, a fall event is detected. Our algorithm, coupled with accelerometers and gyroscopes, reduces both false positives and false negatives, while improving fall detection accuracy. In addition, our solution features low computational cost and real-time response.
\end{abstract}

\section{Introduction}

Falls are detrimental events for the aged population. According to [10], more than $33 \%$ of people age 65 years or older have one fall per year. Fall risk is also higher for people from special careers such as fire fighting. Hence, reliable fall detection is of great importance.
Falls are a common issue, but they are difficult to define rigorously. Since falls are usually characterized by larger acceleration compared with ADL, existing solutions mainly use accelerometers for detection [8]. However, focusing only on large acceleration can result in many false positives from fall-like activities such as sitting down quickly and jumping.

Some fall detection algorithms also assume that falls often end with a person lying prone horizontally on the floor. These kinds of systems use change of body orientation as an indicator for falls [4]. But, they are less effective when a person is not lying horizontally, e.g. a fall may happen on stairs.

To improve activity recognition accuracy, a large body of work uses complex inference techniques like hidden Markov models to analyze acceleration data [6], but they use excessive amounts of computational resources and do not always meet real-time constraints. Such methods are inappropriate for fall detection because fast response is essential. In addition, fall activity patterns are particularly difficult to obtain for training such systems.

Our solution divides human activities into two categories: static postures and dynamic transitions between these postures. We define falling as an unintentional transition to the lying posture. Using two tri-axial accelerometers at different body locations, our system can recognize four kinds of postures: standing, sitting, bending, and lying. This is more accurate than only using body orientation information. To determine whether a transition is intentional, our system measures not only linear acceleration, but also angular velocity with gyroscopes. By using both accelerometer-derived posture information and gyroscopes, our fall detection algorithm is more accurate than existing methods. Moreover, our solution has low computational cost and fast response.

The rest of the paper is divided into four sections. Sec. 2 
gives an overview of existing fall detection systems. Sec. 3 proposes our fall detection solution. The evaluation of our solution is presented in Sec. 4. Sec. 5 concludes the paper and gives directions for future work.

\section{State of the Art}

Existing fall detection solutions can be divided into two classes. The first class only analyzes acceleration to detect falls. Prado [13] [5] used a four-axis accelerometer located at the height of the sacrum to detect falls. Mathie [9] used a single, waist-mounted, tri-axial accelerometer to detect falls. Lindemann [8] integrated a tri-axial accelerometer into a hearing aid housing, and used thresholds for acceleration and velocity to decide if falls happen. Kangas [7] studied acceleration of falls and ADL from the waist, wrist, and head, and showed that measurements from the waist and head were more useful for fall detection. Bourke [3] placed two tri-axial accelerometers at the trunk and thigh, and derived four thresholds, upper and lower thresholds for both the trunk and thigh. Exceeding any of the four thresholds indicated a fall had occurred. The problem with this kind of method is that other ADL such as sitting down quickly and jumping also features large vertical acceleration. Therefore, only using acceleration for fall detection causes many false positives.

A second class of solutions utilize both acceleration and body orientation information to detect falls. Noury [12] developed a fall detector consisting of three sensors: a tilt sensor to monitor body orientation, a piezoelectric accelerometer to monitor vertical acceleration shock, and a vibration sensor to monitor body movements. Noury [11] developed a sensor with two orthogonally oriented accelerometers and used this sensor to monitor the inclination and inclination speed to detect falls. Chen [4] looked at the change in body orientation during an impact to monitor falls. Body orientation can help improve the fall detection accuracy, but using one single device can only monitor the orientation of the trunk, more posture information cannot be collected using this kind of method. Bourke [2] developed a thresholdbased fall detection algorithm using a bi-axial gyroscope sensor. They put the gyroscope at the sternum, and measured angular velocity, angular acceleration, and change in trunk angle to detect falls.

Besides the two main kinds of fall detection solutions outlined above, complex inference techniques are also utilized to improve activity recognition accuracy. Raghu et al. [6] attached five accelerometers to a jacket, and performed activity recognition by using hidden Markov models to analyze acceleration data. Their method needs activity patterns and significant computation, so it is not appropriate for fall detection.

Some commercial health monitoring products such as

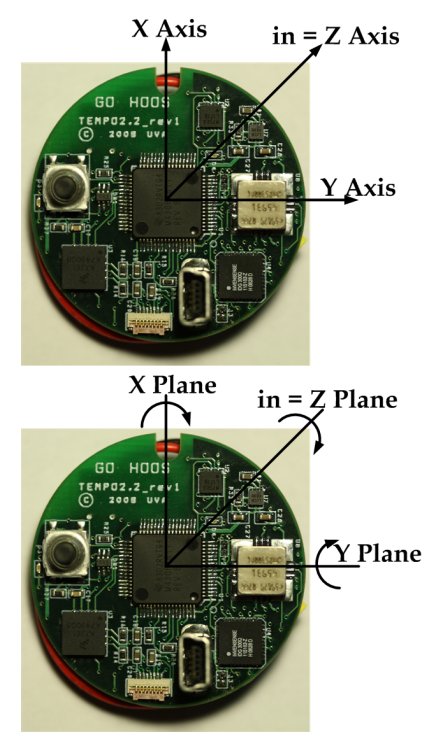

(a)

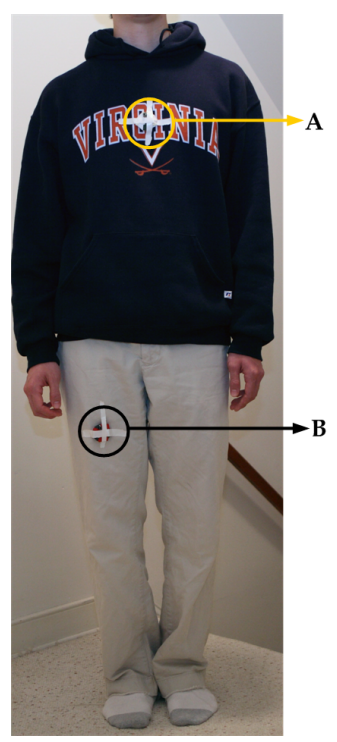

(b)
Figure 1: (a) The TEMPO 3.0 sensor node; (b) The placement of two TEMPO 3.0 nodes

Philips' Lifeline [1] use a help button to issue medial alerts. However, when a really serious fall happens, people may not be able to push the button. Therefore, improving the accuracy of automatic fall detection is of great importance.

\section{Methodology}

\subsection{Data Acquisition}

Since our solution measures both acceleration and angular velocity to detect falls, we chose to use the TEMPO (Technology-Enabled Medical Precision Observation) 3.0 sensor nodes.

The TEMPO 3.0 node includes a tri-axial accelerometer and a tri-axial gyroscope as shown in Fig. 1(a). The triaxial accelerometer, an MMA7261QT made by Freescale Semiconductor, can monitor acceleration within a range of $\pm 10 \mathrm{~g}$. The tri-axial gyroscope consists of an InvenSensce IDG-300 dual-axis gyroscope and an Analog Devices ADXRS300 Z-axis gyroscope. The IDG-300 can monitor angular velocity between $\pm 500 \%$. The ADXRS300 can monitor angular velocity between $\pm 300 \%$. The sensors are controlled by an TI MSP430F1611 microcontroller. The sampling rate is set to $120 \mathrm{~Hz}$, a bandwidth exceeding the characteristic response of human movement.

Considering that most postures have different angles between the trunk and upper legs, the sensor nodes are attached on the chest (Node $A$ ) and thigh (Node $B$ ) as shown in Fig. 1(b). For the following experiments, three male subjects in their 20 s engaged in a battery of tests designed to 
simulate ADL, falls, and fall-like activities. To conduct these tests, four continuous data sets were collected from each subject with approximately 5 seconds spent in each activity: ADL (walk on stairs, walk, sit, jump, lay down, run, run on stairs), fall-like motions (quickly sit-down upright, quickly sit-down reclined), flat surface falls (fall forward, fall backward, fall right, fall left), inclined falls (fall on stairs). All fall data was taken on hard surfaces. In addition, static posture data (standing, bending, sitting, and lying) was collected from a single subject to explore the accuracy of the proposed posture recognition algorithm. The following sections present some of the collected data and discuss the efficacy of the proposed fall detection solution.

\subsection{The Fall Detection Algorithm}

Our fall detection solution can be divided into three steps: activity intensity analysis, posture analysis, and transition analysis.

The data collected are segmented into one second intervals. If the change of sensor readings within an interval falls into the region specified in Line 2 of Algorithm 1, it is classified as a static posture. Otherwise, a dynamic transition is assumed. For static segments, the accelerometer readings are used to determine specific postures, including standing, bending, sitting, and lying. If the posture of a static segment is determined to be lying, we examine whether the transition to the lying posture was an intentional movement by examining the previous 5 seconds of data. If the transition was unintentional, it is flagged as a fall. The three-phase fall detection process is shown in Algorithm 1. Sec. 3.2.1 to Sec. 3.2.3 explain the process in more detail.

\subsubsection{Dynamic Transitions vs. Static Postures}

As shown in Fig. 1(b), two TEMPO 3.0 nodes, $A$ and $B$, are attached on the chest and right thigh, respectively. Using

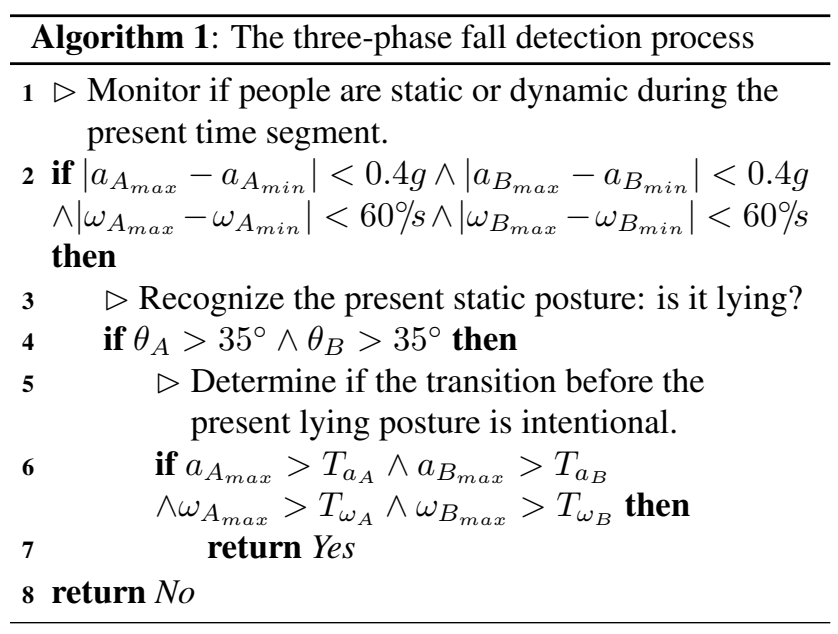

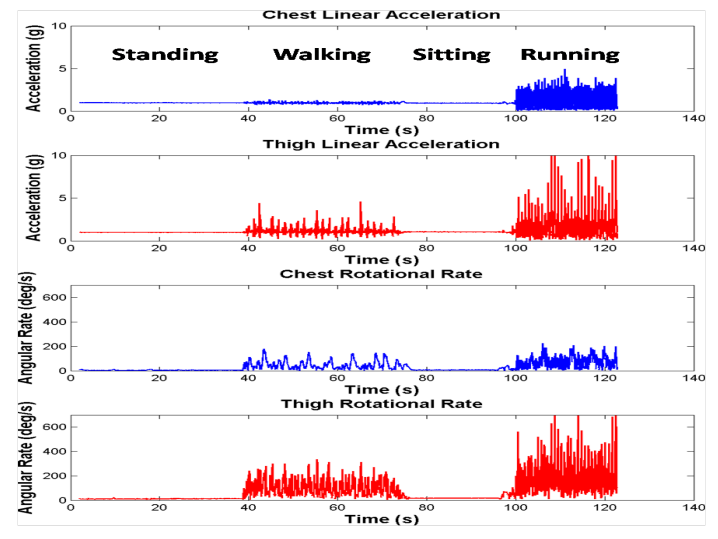

Figure 2: The linear acceleration and rotational rate of the trunk and thigh for standing, walking, sitting, and running

these two nodes we can get both the linear acceleration and rotational rate of the trunk and thigh:

$$
\begin{aligned}
a_{A} & =\sqrt{a_{A_{x}}^{2}+a_{A_{y}}^{2}+a_{A_{z}}^{2}} \\
a_{B} & =\sqrt{a_{B_{x}}^{2}+a_{B_{y}}^{2}+a_{B_{z}}^{2}} \\
\omega_{A} & =\sqrt{\omega_{A_{x}}^{2}+\omega_{A_{y}}^{2}+\omega_{A_{z}}^{2}} \\
\omega_{B} & =\sqrt{\omega_{B_{x}}^{2}+\omega_{B_{y}}^{2}+\omega_{B_{z}}^{2}}
\end{aligned}
$$

Here, $a_{A}$ and $a_{B}$ are the chest and thigh vector magnitude linear acceleration, respectively. $\omega_{A}$ and $\omega_{B}$ serve as measures of aggregate rotational rate as derived in [2].

Fig. 2 shows the linear acceleration and rotational rate readings from nodes $A$ and $B$ for typical standing, walking, sitting and running. From this figure we can see that the acceleration amplitude for stationary postures is smaller than $0.40 \mathrm{~g}$, and the rotational rate amplitude for stationary postures is smaller than $60 \%$. Using these thresholds we can separate static postures from dynamic transitions quickly and accurately.

\subsubsection{Posture Recognition}

As shown in Algorithm 1 when a static posture is detected, it must be determined whether a person is lying prone. Since the posture is static, $a_{A}$ and $a_{B}$ in (1) should always be near the gravitational constant: $1.0 \mathrm{~g}$. Then we can calculate the angle between the trunk and the gravitational vector, $\theta_{A}$, and the angle between the thigh and the gravitational vector, $\theta_{B}$ :

$$
\theta_{A}=\arccos \frac{a_{A_{x}}}{g}, \theta_{B}=\arccos \frac{a_{B_{x}}}{g}
$$

Fig. 3 shows $\theta_{A}$ and $\theta_{B}$ for four kinds of static postures: standing, bending, sitting, and lying. These postures are characterized by different inclination angles of the trunk and thigh. These angles are specified in Table 1. 


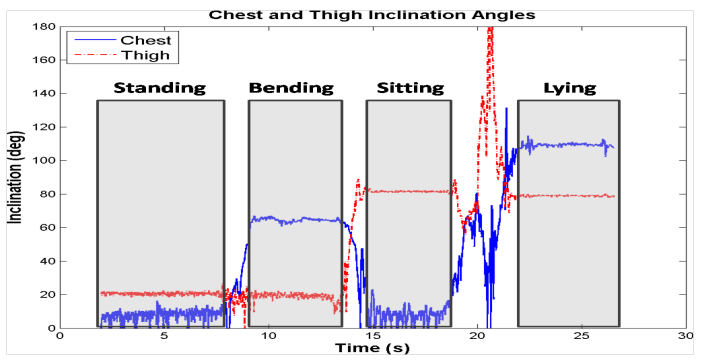

Figure 3: The inclination angles of the trunk and thigh for four static postures: standing, bending, sitting, and lying

\begin{tabular}{c|c|c}
\hline$\theta_{A}(\mathrm{deg})$ & $\theta_{B}(\mathrm{deg})$ & Posture \\
\hline$<35$ & $<35$ & Standing \\
\hline$>35$ & $<35$ & Bending \\
\hline$<35$ & $>35$ & Sitting \\
\hline$>35$ & $>35$ & Lying \\
\hline
\end{tabular}

Table 1: Postures are determined by different inclination angles of the trunk and thigh

\subsubsection{Intentional vs. Unintentional}

An unintentional transition to a lying posture is regarded as a fall, and it features large accelerometer and gyroscope readings. We differentiate intentional and unintentional transitions by applying thresholds to peak values of acceleration ( $a$ ) and angular rate $(\omega)$ from two nodes, $A$ and $B$, as shown in Line 6 of Algorithm 1.

The acceleration and rotational rate were compared over ADL and fall datasets to determine $T_{a_{A}}, T_{a_{B}}, T_{\omega_{A}}$ and $T_{\omega_{B}}$. Fig. 4 shows the linear acceleration and rotational rate of the chest and thigh for ADL. Activities include going upstairs, walking, sitting down deliberately, jumping, lying down deliberately, running, and going downstairs quickly.

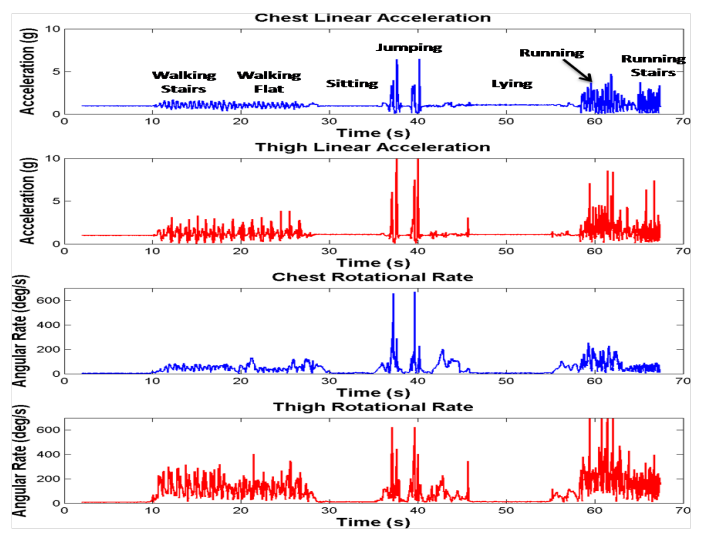

Figure 4: The linear acceleration and rotational rate of the trunk and thigh for ADL
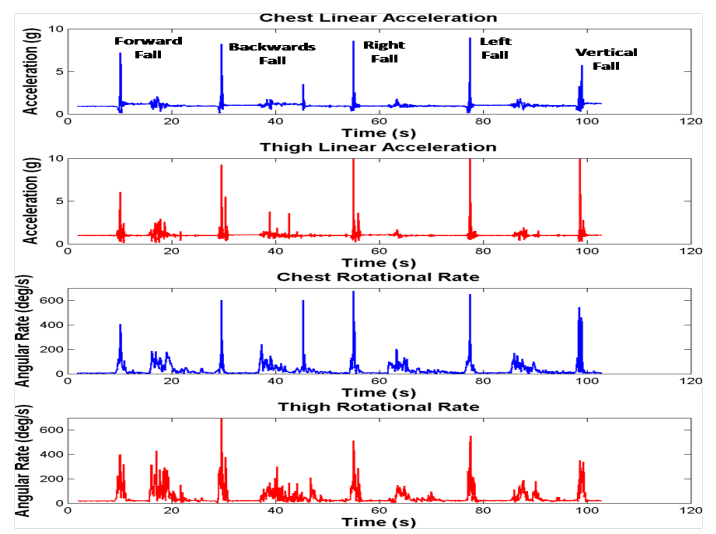

Figure 5: The linear acceleration and rotational rate of the trunk and thigh for falls

Fig. 5 illustrates the acceleration and rotational rate of typical forward, backward, rightward, leftward, and vertical falls. Inspection of these figures reveals that falls and vigorous daily activities such as jumping, running, going upstairs/downstairs quickly are characterized by larger acceleration and rotational rate. Using $T_{a_{A}}=3.0 \mathrm{~g}, T_{a_{B}}=2.5 \mathrm{~g}$, $T_{\omega_{A}}=200 \%$ and $T_{\omega_{B}}=340 \%$ can distinguish these abrupt transitions from normal gentle activities. It should be noted, however, that such thresholds are influenced by a person's profile (e.g. height, weight, age). More work is needed to find these relationships.

\section{Evaluation}

In this section we evaluate the accuracy of our fall detection method by: first studying two special cases, then running a continuous monitoring test.

\subsection{Special Case Study}

As mentioned in Sec. 2, existing methods monitor the acceleration and/or body orientation to detect falls. Here we show two activities that cannot be distinguished from these two variables alone, but can be distinguished using our method.

\subsubsection{Sit Down Fast}

Some existing acceleration-based fall detection systems [13] [5] [9] [7] [3] only use acceleration to differentiate falls from ADL. However, some activities like sitting down fast also feature large vertical acceleration. Fig. 6 shows the acceleration and rotational rate of the trunk and thigh for sitting fast. Both $a_{A_{\max }}$ and $a_{B_{\max }}$ are larger than thresholds used in [13], [5], [9], [7], and [3]. Therefore, sitting down fast is not distinguishable from a typical fall. 

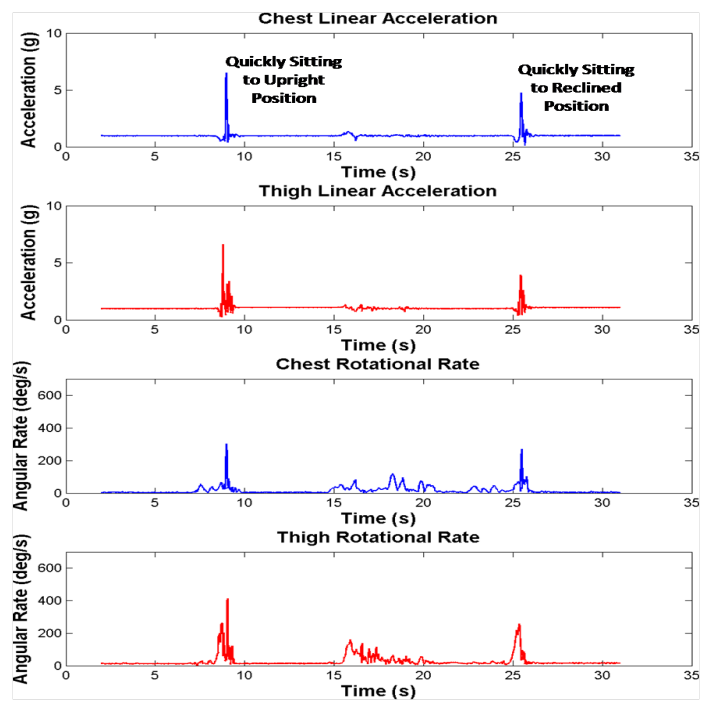

Figure 6: The linear acceleration and rotational rate of the trunk and thigh for sitting fast, ending postures are sitting straight and leaning back

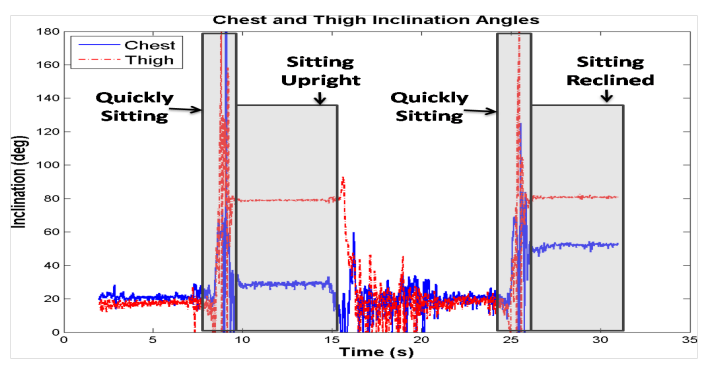

Figure 7: The inclination angles of the trunk and thigh for sitting fast, ending postures are sitting straight and leaning back

Using accelerometer-derived posture information, gyroscopes, and our algorithm, we can distinguish sitting down fast from falls. If the ending posture is sitting straight, according to Table 1, and the inclination angles of the trunk and thigh are consistent with the data shown in Fig. 7, we can determine that the transition is not a fall according to Line 4 of Algorithm 1. If the ending posture is leaning backward, we can determine that the transtion is not a fall according to Line 6 of Algorithm 1.

\subsubsection{Fall on Stairs}

Horizontal body orientation is used as a sign of falls in [12] and [11], but this triggers false negatives when falls happen on non-horizontal planes such as with stairs. In [4] and [2], the trunk inclination change is used to detect falls, but this method triggers false positives when people bend down quickly.

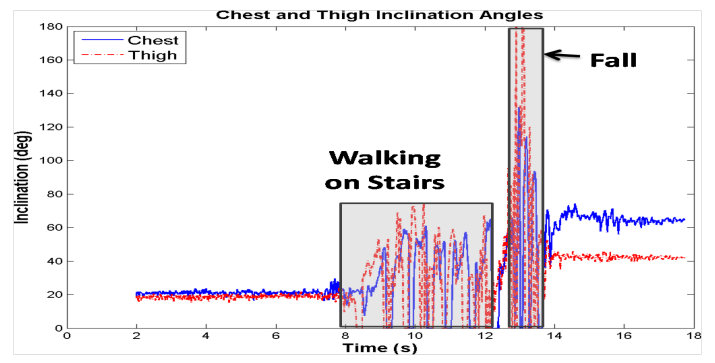

Figure 8: The inclination angles of the trunk and thigh for falling on stairs
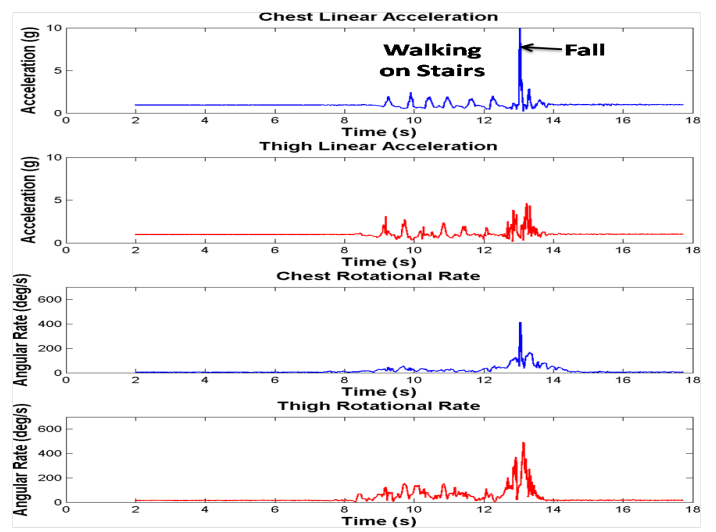

Figure 9: The linear acceleration and rotational rate of the trunk and thigh for falling on stairs

As described in Sec. 3.2.2, our solution examines the inclination angles of the trunk and thigh to extract posture information. This technique is more accurate than only attaching one node on the chest or waist to detect body orientation. Fig. 8 shows the inclination angles of the trunk and thigh for a typical fall on stairs. Both $\theta_{A}$ and $\theta_{B}$ are larger than $35^{\circ}$. According to Table 1, the posture would be classified as lying. Fig. 9 demonstrates that Algorithm 1 can recognize falling on stairs accurately using thresholds mentioned in Sec. 3.2.3.

\subsection{Continuous Monitoring}

In this section we monitor activities continuously, and show that our solution is effective for typical activities. The activities include ADL, fall-like motions (e.g. sitting down fast, jumping, going up/downstairs, stumbling, lying down), and different kinds of falls (e.g. falling forward/backward/leftward/rightward/vertically). Fig. 10(a) shows the false negatives performance of our algorithm. Our method can detect typical falls and falling on stairs very accurately. However, it is not as effective when people fall against walls ending with a sitting position. The sensitivity of our algorithm is $91 \%$ from 70 records. Fig. 10(b) shows 


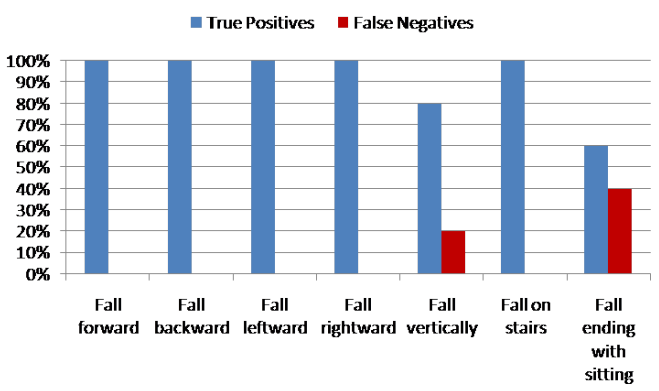

(a)

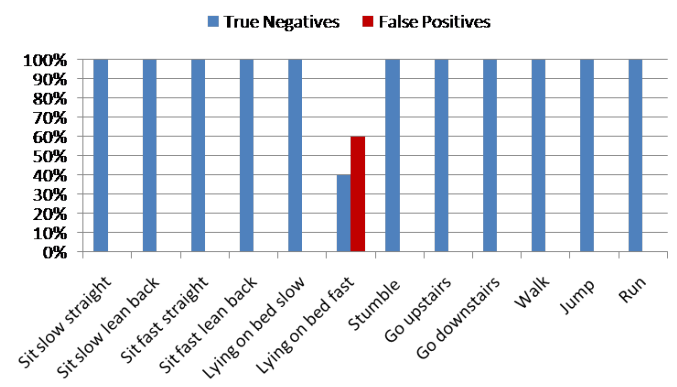

(b)

Figure 10: (a) False negatives performance (sensitivity); (b) False positives performance (specificity)

the false positives performance of our algorithm. It can exclude most of the fall-like activities. However, quickly lying down sometimes triggers false positives. The specificity is $92 \%$ from 72 records.

\subsection{Speed Analysis}

Our solution does not need complex computation, so the detection process can be implemented on an simple, bodyworn aggregator like a PDA or cellphone. Moreover, the algorithm only needs to buffer 6 seconds of data, which makes it possible for our solution to respond quickly.

\section{Conclusion and Future work}

In this work, we present a fall detection algorithm that can reduce both false positives (e.g. sitting down fast) and false negatives (e.g. falling on stairs) by using gyroscopes and accelerometer-derived posture information. It also features low computational cost and fast response.

However, our method has difficulties in differentiating jumping into bed and falling against wall with a seated posture. To distinguish these activities, context information (environmental/physiological) can be exploited in future work.

\section{References}

[1] http://www.lifelinesys.com/content/lifelineproducts/classic-pendant.jsp.

[2] A. K. Bourke and G. M. Lyons. A threshold-based falldetection algorithm using a bi-axial gyroscope sensor. Medical Engineering and Physics, 30:84-90, Jan 2008.

[3] A. K. Bourke, J. V. O'Brien, and G. M. Lyons. Evaluation of a threshold-based tri-axial accelerometer fall detection algorithm. Gait and Posture, 26:194-199, 2007.

[4] J. Chen, K. Kwong, D. Chang, J. Luk, and R. Bajcsy. Wearable sensors for reliable fall detection. In Proceedings of the 27th Annual International Conference of the IEEE EMBS, pages 3551-3554, Shanghai, China, Sept 2005. IEEE.

[5] A. Díaz, M. Prado, L. M. Roa, J. Reina-Tosina, and G. Sánchez. Preliminary evaluation of a full-time falling monitor for the elderly. In Proceedings of the 26th Annual International Conference of the IEEE EMBS, pages 2180 2183, San Francisco, USA, Sept 2004. IEEE.

[6] R. K. Ganti, P. Jayachandran, T. F. Abdelzaher, and J. A. Stankovic. SATIRE: A software architecture for smart attire. In Proceedings of MobiSys'06, Uppsala, Sweden, June 2006. ACM Press.

[7] M. Kangas, A. Konttila, I. Winblad, and T. Jämsä. Determination of simple thresholds for accelerometry-based parameters for fall detection. In Proceedings of the 29th Annual International Conference of the IEEE EMBS, pages 13671370, Lyon, France, Aug 2007. IEEE.

[8] U. Lindemann, A. Hock, M. Stuber, W. Keck, and C. Becker. Evaluation of a fall detector based on accelerometers: A pilot study. Medical and Biological Engineering and Computing, 43(5):548-551, Oct 2005.

[9] M. J. Mathie, J. Basilakis, and B. G. Celler. A system for monitoring posture and physical activity using accelerometers. In Proceedings of the 23rd Annual International Conference of the IEEE EMBS, pages 3654-3657, Istanbul, Turkey, Oct 2001. IEEE.

[10] N. Noury. A smart sensor for the remote follow up of activity and falldetection of the elderly. In Proceedings of the 2 nd International IEEE EMBS Special Topic Conference on Microtechnologies in Medicine and Biology, pages 314-317, 2002.

[11] N. Noury, P. Barralon, G. Virone, P. Boissy, M. Hamel, and P. Rumeau. A smart sensor based on rules and its evaluation in daily routines. In Proceedings of the 25th Annual International Conference of the IEEE EMBS, pages 3286-3289, Cancun, Mexico, Sept 2003. IEEE.

[12] N. Noury, T. Hervé, V. Rialle, G. Virone, E. Mercier, G. Morey, A. Moro, and T. Porcheron. Monitoring behavior in home using a smart fall sensor and position sensors. In Proceedings of the 1st International IEEE EMBS Special Topic Conference on Microtechnologies in Medicine and Biology, pages 607-610, Lyon, France, Oct 2000. IEEE.

[13] M. Prado, J. Reina-Tosina, and L. Roa. Distributed intelligent architecture for falling detection and physical activity analysis in the elderly. In Proceedings of the 2 nd Joint EMBS/BMES conference, pages 1910-1911, Houston, USA, Oct 2002. IEEE. 